\title{
Bioguided isolation of cytotoxic compounds against melanoma cells from Carissa spinarum $L$.
}

\author{
A Alqathama 1, 2, A Bader 2, P Khondkar 1, S Gibbons 1, J Prieto 1 \\ 1UCL, London, United Kingdom \\ 2Umm AIQura University, Makkah, Saudi Arabia \\ Congress Abstract
}

Malignant melanoma is the most aggressive form of skin cancer. As natural products are an important source of new anti-cancer lead compounds, the aim of this study is to screen selected Saudi medicinal plants for anti-melanoma activity by evaluating their effects on cell proliferation, cell cycle profile and apoptosis induction, as well as to characterize the active principle compounds.

Based on our preliminary results [1], several extracts of selected Saudi medicinal plants were active against melanoma cell lines. Among them Carissa spinarum L. (synonym Carissa edulis (Forssk.) Vahl) was one of the most promising plants. The active extract of this plant was subjected to VLC, preparative TLC and the isolated compounds were characterised by NMR and MS. The cytotoxic effects on A375 melanoma cells of the extracts were determined by Sulforhodamine B staining assay. Further mechanistic studies were performed namely caspase $3 / 7$ activity and cell cycle analysis.

The hexane extract retained most of the cytotoxicity (IC50 $40 \mu \mathrm{g} / \mathrm{mL}$ ), followed by the chloroform (IC50 $47 \mu \mathrm{g} / \mathrm{mL}$ ) and methanolic (IC50 > $100 \mu \mathrm{g} / \mathrm{mL}$ ) extracts.

Cell cycle analysis showed that the hexane extract was able to induce an arrest at $S$ phase and this was accompanied by the induction of caspase $3 / 7$ activities. Its fractionation using VLC (normal phase, Silica gel) afforded 11 fractions. All fractions were screened for cytotoxicity and fraction no. 11 (eluted with $100 \%$ ethyl acetate) was one of the most active ones. Preparative TLC (Silica gel 60 using hexane:ethyl acetate as solvent system) was used to purify the fraction, which afforded 2 compounds (IC50 $\approx 20 \mu \mathrm{g} / \mathrm{mL}$ ). Their spectral and spectroscopic data are consistent with ursolic acid and a closely related compound.

References:

[1] Alqathama A, Prieto JM, Anti-migratory and cytotoxic properties of medicinal plants against melanoma. Eur J Cancer 2014; 50: S198 